\title{
Pan-cancer analysis identifies FAM49B as an immune- related prognostic maker for hepatocellular carcinoma
}

\author{
Feng $\mathrm{Xu}^{1^{*}}$, Jionghuang $\mathrm{Chen}^{2^{*}}$, Dihua Huang ${ }^{3^{凶}}$ \\ 1. Department of General Surgery, Shaoxing People's Hospital (Shaoxing Hospital, Zhejiang University School of Medicine), Shaoxing, Zhejiang, China. \\ 2. Department of General Surgery, Sir Run Run Shaw Hospital, Zhejiang University School of Medicine, Hangzhou, China. \\ 3. Department of Endocrinology, Shaoxing People's Hospital (Shaoxing hospital, Zhejiang University School of Medicine), Shaoxing, Zhejiang, China. \\ *These authors contributed equally to this work.
}

$\square$ Corresponding author: Dihua Huang, Department of Endocrinology, Shaoxing People's Hospital (Shaoxing hospital, Zhejiang University School of Medicine), Shaoxing, Zhejiang, China. E-mail: huangdh@usx.edu.cn.

() The author(s). This is an open access article distributed under the terms of the Creative Commons Attribution License (https://creativecommons.org/licenses/by/4.0/). See http://ivyspring.com/terms for full terms and conditions.

Received: 2021.07.27; Accepted: 2021.11.16; Published: 2022.01.01

\begin{abstract}
Family with sequence similarity 49, member B (FAM49B) is highly expressed in many tumors, its role in malignant tumors especially in hepatocellular carcinoma $(\mathrm{HCC})$ remains uncertain. We first evaluated the expression, clinical features, and prognostic value of FAM49B using RNA-seq and clinical data from The Cancer Genome Atlas. We further assessed the role of FAM49B in the tumor immune microenvironment. The correlation of FAM49B with the sensitivity of 192 anti-cancer drugs was analyzed using data from Genomics of Drug Sensitivity in Cancer database. qRT-PCR assay was used to validate the expression of FAM49B in HCC. FAM49B was expressed at high levels in most tumor types, including HCC. High FAM49B expression predicted poor survival in patients with HCC. We also found that FAM49B expression was negatively associated with the infiltration levels of immune killer cells, including NK cells, and positively associated with immunosuppressive cells, including Tregs and Central Memory $T$ cell $(\mathrm{Tcm})$, in HCC. In addition, FAM49B expression was positively associated with immune checkpoints, immune regulation genes, $\mathrm{MHC}$ genes, chemokines and chemokine receptors. Patients with evaluated expression of FAM49B might be resistant to several anti-cancer drugs. Our results suggest that FAM49B is a potential prognostic biomarker for HCC. FAM49B play a potential key role in regulating tumor immune microenvironment and anti-tumor drug tolerance.
\end{abstract}

Key words: FAM49B, TCGA, hepatocellular carcinoma, immune microenvironment, prognostic biomarker

\section{Introduction}

In recent years, significant progress has been made in cancer-related treatment technologies in hepatocellular carcinoma (HCC). However, the survival rate for HCC is still very low $[1,2]$. Studies have shown that there is a certain relationship between tumor immune microenvironment and tumor progression in HCC [3-5]. The immunosuppressive microenvironment has a poor effect on the prognosis of patients with HCC. Thus, it is urgent for ameliorating the prognosis of HCC patients to detect immunosuppressive status-related prognostic marker and potential drug targets.

Family with sequence similarity 49 , member B (FAM49B), localized on chromosome 8q24, encodes for a $37-\mathrm{kDa}$ protein, was previously reported to involve progression of several diseases, including tumor progression [6]. For example, FAM49B was up-regulated and promoted proliferation and metastasis of gallbladder cancer cell [7].

Suppressing the immune checkpoint and immunosuppressive cells, activating $\mathrm{T}$ cells and NK cells, provides a new idea for tumor immunotherapy [8]. Interestingly, researches have reported that FAM49B could inhibit $\mathrm{T}$ cell activation by inhibiting Rac activity and regulating cytoskeleton reorganization [9]. These reports indicate that FAM49B may play an essential role in tumor progression. However, the role of FAM49B in HCC remains uncertain.

In our study, we comprehensively analyzed the role of FAM49B in HCC and pan-cancer, including the 
expression, prognostic value, DNA methylation, and copy number alteration (CNA) alteration of FAM49B. The correlation between FAM49B expression and infiltration level of immune cell and immune regulation-related genes were further evaluated. The sensitiveness of anti-cancer drugs was assessed for patients with high FAM49B expression. Our results provide novel insights into the functional role of FAM49B in HCC, highlighting a potential mechanism whereby FAM49B influences the tumor immune microenvironment (TIME), as well as prognosis of tumor patients.

\section{Materials and methods}

\section{Data collection}

The RNA-seq and clinical data of TCGA, CCLE, and GTEx were downloaded from UCSC XENA website (https://xenabrowser.net/datapages/). The detailed criteria for the inclusion and exclusion of patients enrolled into the current study can be found in the website of TCGA (https://www.cancer.gov/ about-nci/organization/ccg/research/structuralgenomics/tcga/studied-cancers). Surgical resection of biopsy biospecimens were collected from patients diagnosed with hepatocellular carcinoma (HCC), and had not received prior treatment for their disease (ablation, chemotherapy, or radiotherapy). As a part of The Cancer Genome Atlas (TCGA) network, the detailed clinic parameters of enrolled patients with hepatocellular carcinoma were originally published by The Cancer Genome Atlas Research Network [10].

The gene mutation data were obtained from UCSC XENA website. The RNA-seq data were batched and normalized into $\log _{2}(\mathrm{tpm}+0.001)$. The methylation and copy number of FAM49B were downloaded from cBioPortal database (https:// www.cbioportal.org/).

\section{Prognostic analysis}

Univariate Cox regression (uniCox) and Kaplan-Meier analyses were conducted to explore the influence of FAM49B on the survival of patients in pan-cancer using $R$ package "survminer" and "survival". Overall survival (OS), disease-specific survival (DSS), disease-free interval (DFI), and progression-free interval (PFI) of tumor patients were evaluated.

\section{Gene ontology (GO), Kyoto encyclopaedia of genes and genome (KEGG), and Gene set variation analysis (GSVA)}

The top 300 genes most positively associated with FAM49B were selected for enrichment analysis to reflect the function of FAM49B. GO analysis was performed using EnrichGO function in the
"clusterProfiler" R package, KEGG analysis was performed using the EnrichKEGG function of the "clusterProfiler" R package. Gene Set Variation Analysis (GSVA) was conducted using the R package "GSVA" to calculate the pathway score of each sample based on the MSigDB database v7.1 (https:/ / www.gsea-msigdb.org/gsea/msigdb/index.jsp).

\section{Gene set enrichment analysis (GSEA)}

The correlation between FAM49B and all protein-coding mRNAs was analyzed in each tumor from TCGA cohort. The mRNAs correlated with FAM49B (Pearson's correlation coefficient, $p<0.05$ ) were ranked and subjected to GSEA using $R$ package "clusterProfiler".

\section{Tumor immune microenvironment (TIME) analysis}

The R package "ESTIMATE" was used to assess the stromal and immune score of each patient in TCGA cohort. We downloaded the infiltration data of 24 immune cells of 33 tumors from ImmuCellAI database (http://bioinfo.life.hust.edu.cn/ ImmuCellAI\#!/) and conducted the correlation analysis with FAM49B.

\section{Drug resistant analysis}

The IC50, gene expression data, clinical information of 192 drugs and 809 cell lines were downloaded from GDSC (https://www. cancerrxgene.org/). The association between FAM49B expression and IC50 values of 192 drugs were analyzed and Spearman's correlation coefficients were calculated.

\section{Human tissue samples}

10 pairs of HCC tissues and adjacent normal tissues were obtained from HCC patients from 2013 to 2015. The research protocols were approved by the Research Ethics Committee of Sir Run Run Shaw Hospital, School of Medicine, Zhejiang University. All participants gave written consent of their tissue samples and medical information to be analyzed for scientific research.

\section{qRT-PCR}

Total RNA of HCC tissues was isolated and purified by miRNeasy Mini Kit (Qiagen, Maryland, USA). Quantitative PCR (qPCR) analysis of samples was performed using PrimeScript RT Reagent Kit (Takara, Otsu, Japan) following the manufacturer's instructions. ACTB was used as the control gene. The target genes and primers were designed as follows: FAM49B forward 5'-CATGCACAGACCTTGAG CAG-3' and reverse 5'-ATTGCCTCTCGTATTTCG TGG-3', ACTB forward 5'-CATGTACGTTGCTATCC 
AGGC-3' and reverse 5'-CTCCTTAATGTCACGCAC GAT-3'.

\section{Results}

\section{Pan-cancer expression of FAM49B}

We first assessed the expression of FAM49B in 31 tumor types with both tumor and normal tissues using TCGA and GTEx data. Results revealed that FAM49B was over-expressed in 26 of 31 tumors, including BLCA, BRCA, CESC, CHOL, COAD, DLBC, ESCA, GBM, HNSC, KIRC, KIRP, LAML, LGG, LIHC, LUAD, LUSC, OV, PAAD, PRAD, READ, SARC, SKCM, STAD, TGCT, UCEC and UCS, while only lowly expressed in THCA and THYM (Figure 1A). By evaluating the FAM49B expression only in tumor tissues, we observed that FAM49B was highest expressed in LAML and lowest in UVM (Figure 1B). In normal tissues from the GTEx database, results revealed that FAM49B expression was highest in bone marrow and lowest in pancreas (Figure 1C). As for tumor cell lines, we found that FAM49B expression was highest in LAML cell lines using data from the CCLE database (Figure 1D).

We further evaluated the FAM49B expression in paired tumor and adjacent normal tissues. FAM49B was over-expressed in tumor tissues in BLCA, BRCA,
CHOL, COAD, ESCA, HNSC, KIRC, KIRP, LIHC, LUAD, LUSC, STAD, and UCEC (Figure 2A-M). In contrast, FAM49B was lowly expressed in tumor tissues of $\mathrm{KICH}$ and THCA (Figure 2N-O). The expression analysis of FAM49B in LIHC based on tumor grade was performed (Supplementary Figure 1). The results showed that the expression of FAM49B was positively correlates with the tumor grade in LIHC. In addition, qRT-PCR assay confirmed that FAM49B expression was higher in HCC tissues compared to adjacent normal tissues (Figure 2P). Furthermore, the protein level of FAM49B was higher in BRCA, COAD, KIRC, and UCEC using Ualcan database (Figure $3 \mathrm{~A})$.

\section{Gene alteration of FAM49B}

We further evaluated the CNA and methylation status of FAM49B in pan-cancer. For the association between FAM49B and CNA, we found that FAM49B expression were positively correlated with $\mathrm{CNA}$ in LIHC $(r=0.31, p<0.05)$ (Figure 3B). We further proved that the promoter methylation level of FAM49B have little relationship with FAM49B expression $(\mathrm{r}=-0.06, p>0.05)$ in LIHC (Figure 3C). These results indicated that the CNA status mainly contribute to the high expression of FAM49B.

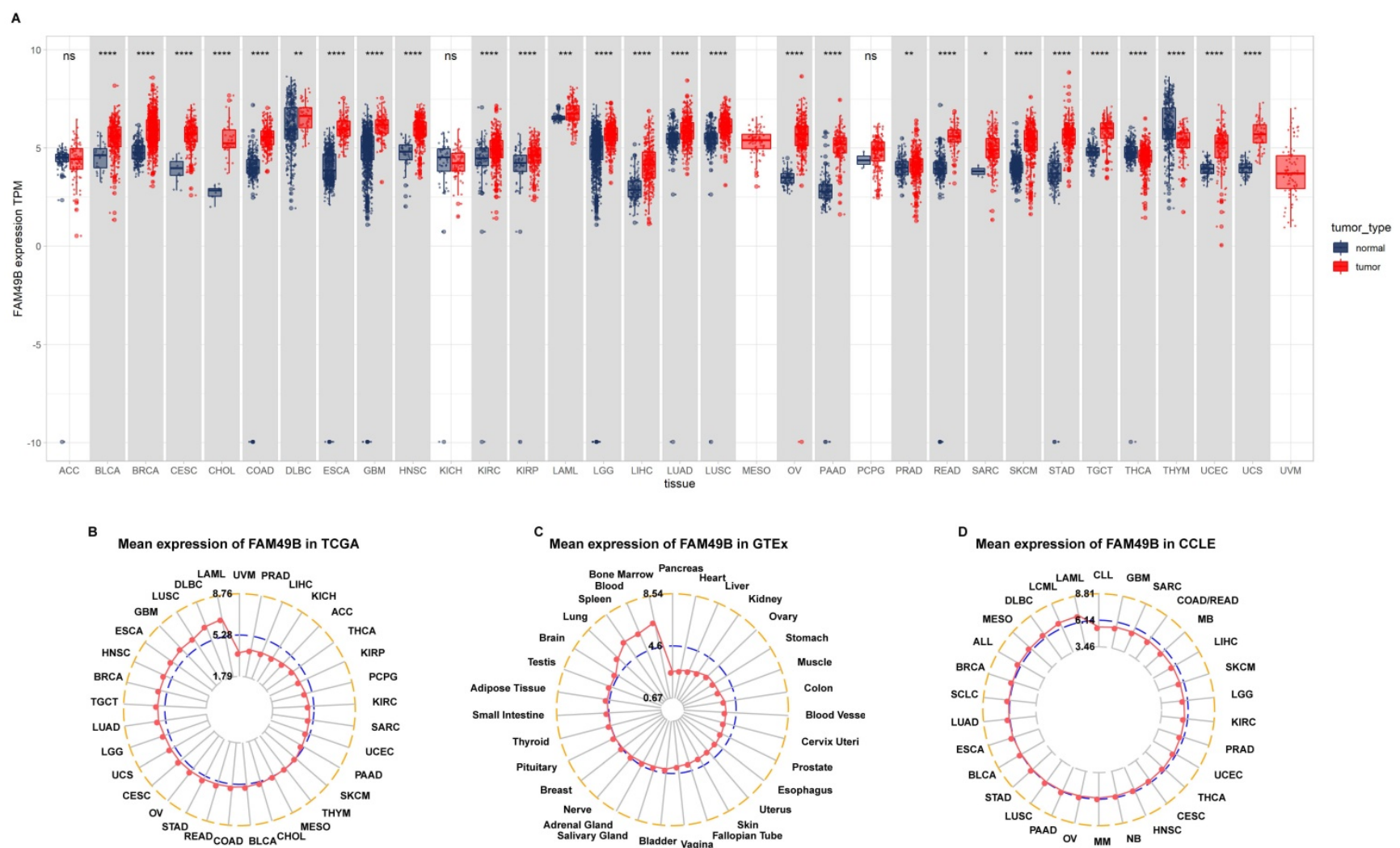

Figure 1. Expression of FAM49B. (A) pan-cancer expression of FAM49B. (B) FAM49B expression in tumor tissues from TCGA cohort. (C) FAM49B expression in normal tissues from GTEx cohort. (D) FAM49B expression in cancer cell lines from CCLE cohort. $* p<0.05, * * p<0.01, * * * p<0.001, * * * * p<0.0001$. 


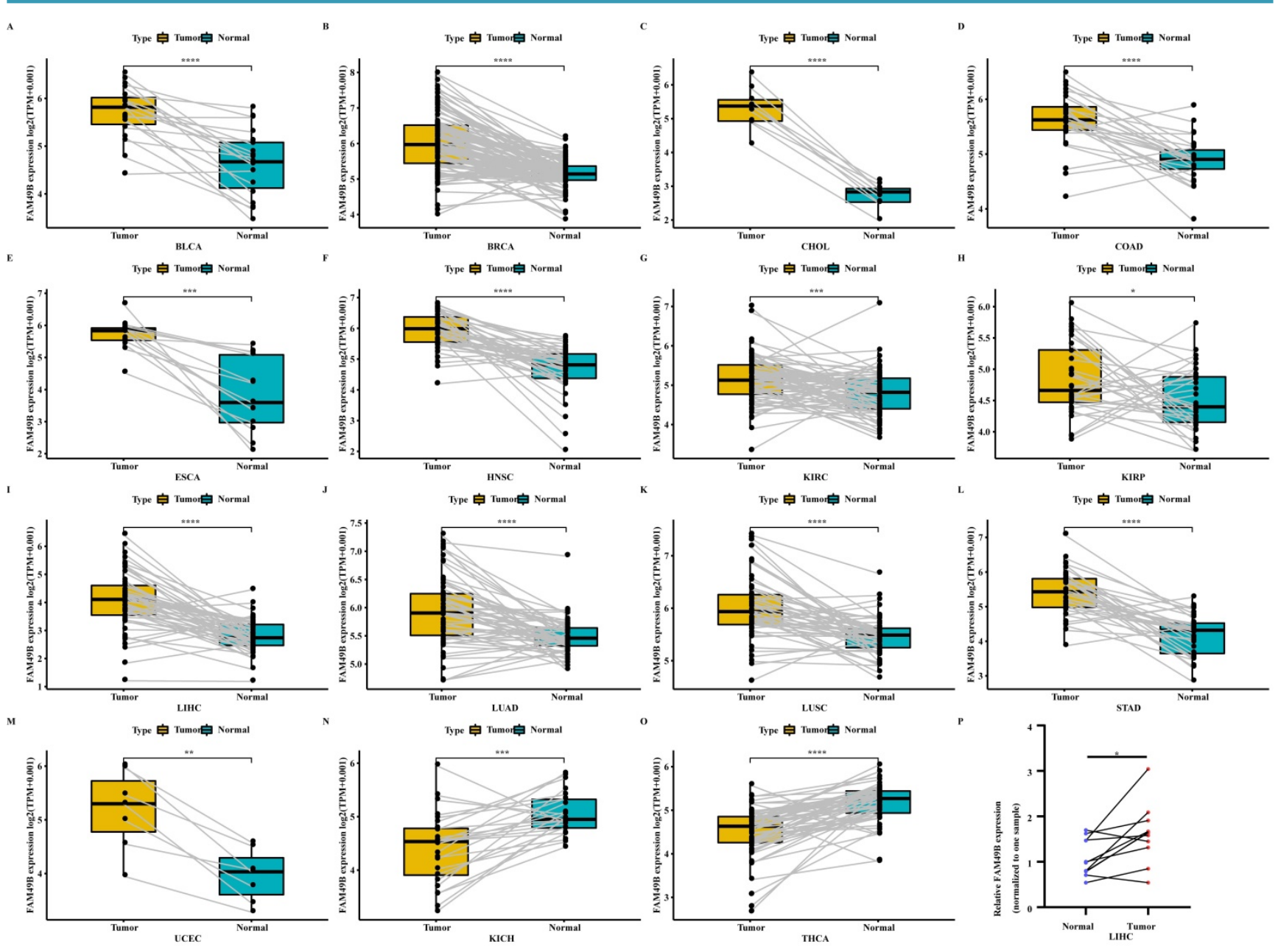

Figure 2. Expression of FAM49B in paired tumor and adjacent normal tissues. (A-O) FAM49B expression in paired tumor and adjacent normal tissues from TCGA in indicated tumor types. (P) qRT-PCR assay evaluates the FAM49B expression in paired tumor and adjacent normal tissues from $\mathrm{HCC}$ patients. $* p<0.05, * * p<0.01, * * * p<$ $0.001, * * * * p<0.0001$.

\section{Prognostic significance of FAM49B}

To evaluate the prognostic role of FAM49B in HCC and pan-cancer, we first conducted uniCox analysis. The uniCox overall survival results indicated that FAM49B was a risk factor in BRCA, HNSC, $\mathrm{KICH}, \mathrm{KIRP}$, LIHC, PAAD, and UVM and protective factor only in SKCM (Figure 4A). For the KaplanMeier analysis of OS, we observed that evaluated FAM49B expression predicted worse OS of patients in 17 tumors in TCGA cohort, such as ACC, BRCA, CESC, HNSC, KICH, KIRP, LAML, LGG, LIHC, LUAD, MESO, PAAD, SARC, THCA, UCEC, UCS, and UVM (Figure 4B).

In addition, the uniCox DSS results indicated that FAM49B was a risk factor in KICH, KIRP, LIHC, PAAD, and UVM (Figure 5A). For DFI results, FAM49B was a risk factor in KIRP and PAAD (Figure 5B). For PFI, a high FAM49B expression predicted shorter PFI times in patients with ACC, HNSC, KICH, KIRP, LIHC, PRAD, and UVM (Figure 5C). These results indicated that FAM49B was a prognostic marker in HCC and other tumors.

\section{Enrichment analyses of FAM49B}

Next, we conducted the GSEA to predict the pathways FAM49B might involve. The genes correlated with FAM49B $(p<0.05)$ were ranked and used to perform GSEA. We analyzed the reactome pathway (GSEA-Reactome) terms using $\mathrm{R}$ package "clusterprofiler" in pan-cancer. The results revealed that FAM49B was enriched in the cell cycle-related pathways (such as Cell Cycle and S Phase) and immune regulation-related pathways (Adaptive Immune System and Innate Immune System) in most tumor types, including BRCA, HNSC, KICH, KIRP, LIHC, and PAAD (Figure 6A-F). The top 20 results of gene ontology (GO), Kyoto encyclopaedia of genes and genome (KEGG), and Gene set variation analysis (GSVA) in FAM49B was also performed (Supplementary Figure 2). 
Expression of FAM49B across cancers (with tumor and nomal samples)
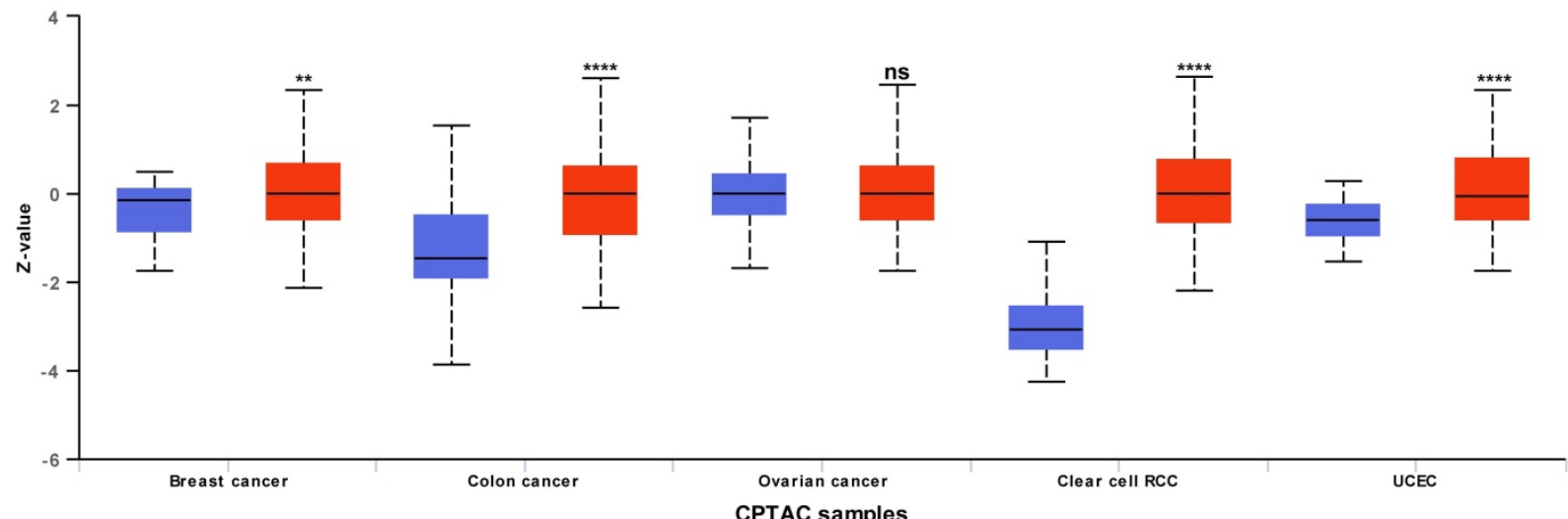

B

label $\bigcirc$ non-significant $\bigcirc$ positive

Ovarian cancer

Clear cell RCC

UCEC CPTAC samples

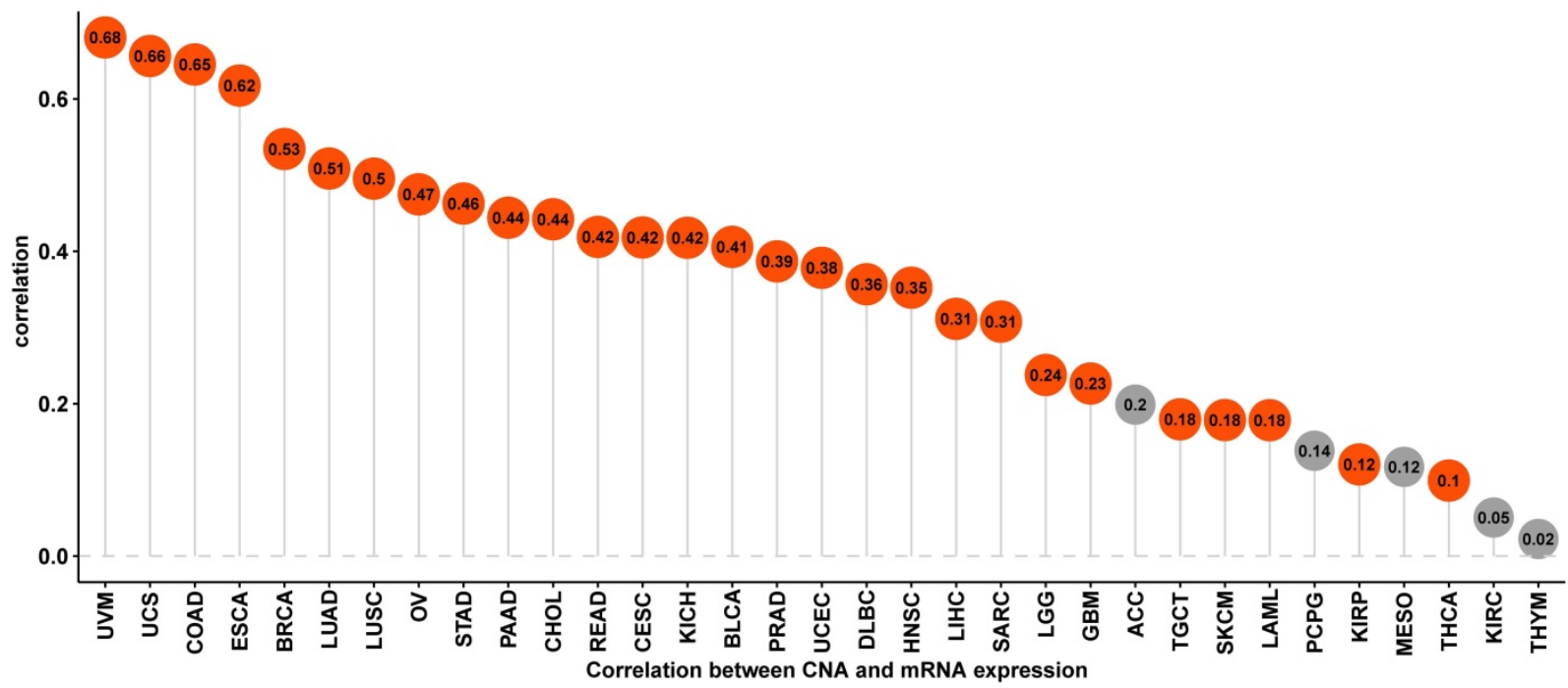

C

label $\bigcirc$ negtive $\bigcirc$ non-significant

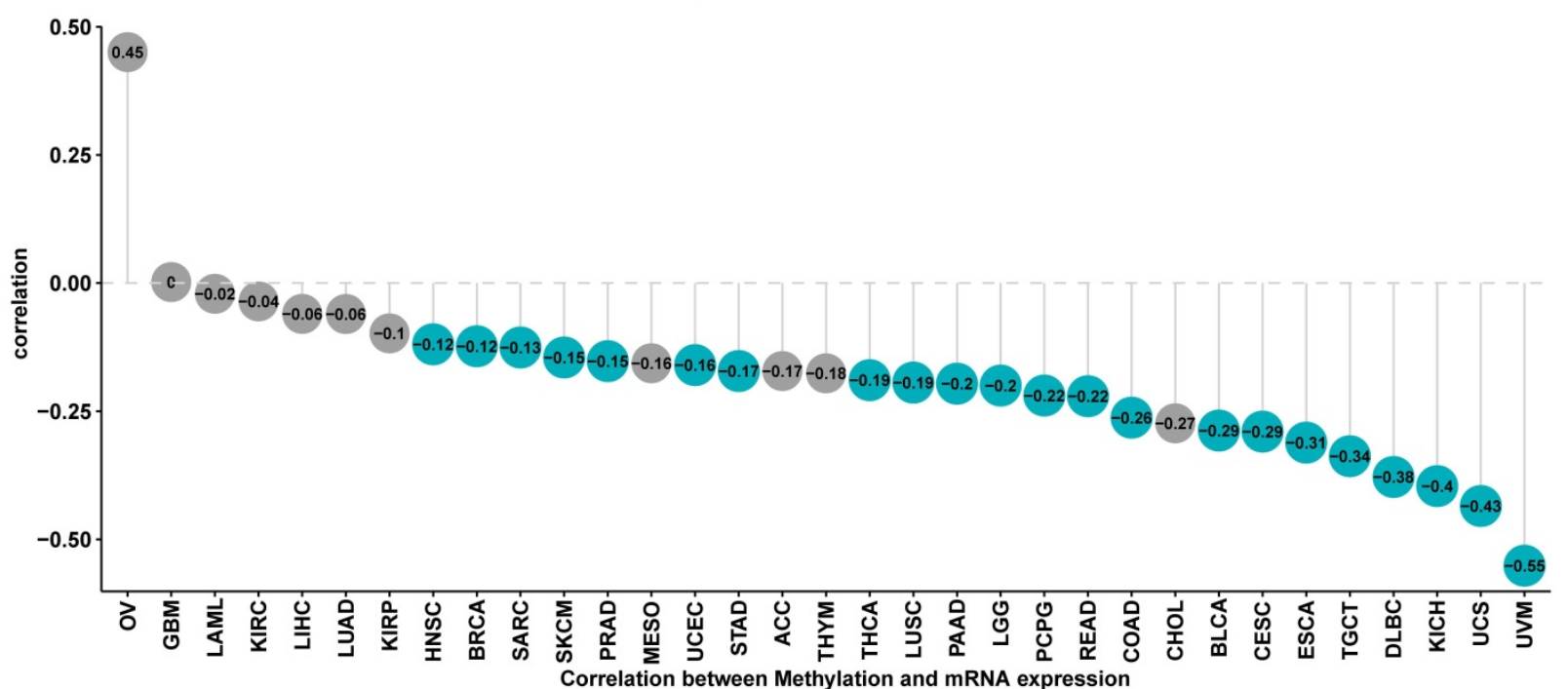

Figure 3. Protein level, CNA and methylation of FAM49B. (A) The protein level of FAM49B in indicated tumor types using Ualcan database. (B) The correlation between FAM49B expression and CNA. (C) The correlation between FAM49B expression and DNA methylation. 
A

$\begin{array}{lcc} & \text { pvalue } & \text { Hazard ratio } \\ \text { ACC } & 0.060 & 1.558(0.982-2.471) \\ \text { BLCA } & 0.613 & 1.052(0.863-1.283) \\ \text { BRCA } & 0.030 & 1.241(1.021-1.508) \\ \text { CESC } & 0.066 & 1.453(0.975-2.163) \\ \text { CHOL } & 0.499 & 0.812(0.444-1.484) \\ \text { COAD } & 0.813 & 0.950(0.619-1.456) \\ \text { DLBC } & 0.529 & 0.734(0.281-1.918) \\ \text { ESCA } & 0.408 & 1.176(0.801-1.724) \\ \text { GBM } & 0.616 & 0.924(0.678-1.259) \\ \text { HNSC } & 0.035 & 1.282(1.017-1.615) \\ \text { KICH } & 0.012 & 5.921(1.488-23.564) \\ \text { KIRC } & 0.628 & 0.949(0.769-1.171) \\ \text { KIRP } & 0.005 & 2.153(1.267-3.659) \\ \text { LAML } & 0.279 & 1.202(0.861-1.678) \\ \text { LGG } & 0.059 & 1.413(0.987-2.021) \\ \text { LIHC } & <0.001 & 1.359(1.141-1.618) \\ \text { LUAD } & 0.070 & 1.231(0.983-1.541) \\ \text { LUSC } & 0.979 & 0.997(0.786-1.264) \\ \text { MESO } & 0.198 & 1.337(0.859-2.079) \\ \text { OV } & 0.247 & 0.930(0.821-1.052) \\ \text { PAAD } & 0.024 & 1.416(1.047-1.914) \\ \text { PCPG } & 0.785 & 0.896(0.408-1.969) \\ \text { PRAD } & 0.249 & 1.708(0.687-4.250) \\ \text { READ } & 0.169 & 0.603(0.293-1.239) \\ \text { SARC } & 0.561 & 1.078(0.836-1.391) \\ \text { SKCM } & 0.005 & 0.798(0.683-0.933) \\ \text { STAD } & 0.353 & 0.886(0.686-1.144) \\ \text { TGCT } & 0.498 & 2.065(0.254-16.813) \\ \text { THCA } & 0.322 & 1.655(0.611-4.478) \\ \text { THYM } & 0.135 & 0.576(0.280-1.187) \\ \text { UCEC } & 0.702 & 1.068(0.762-1.498) \\ \text { UCS } & 0.486 & 1.195(0.724-1.972) \\ \text { UVM } & 0.004 & 1.590(1.164-2.173)\end{array}$

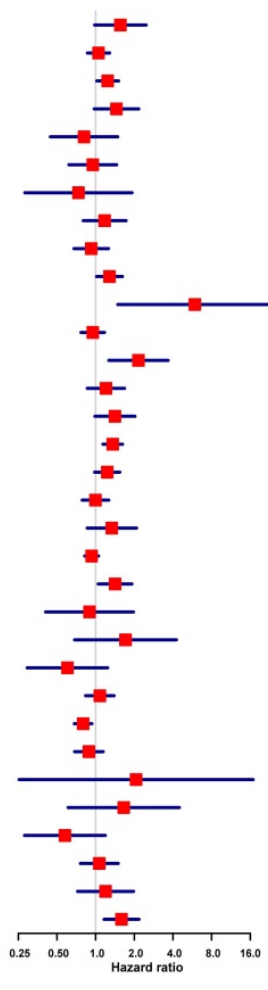

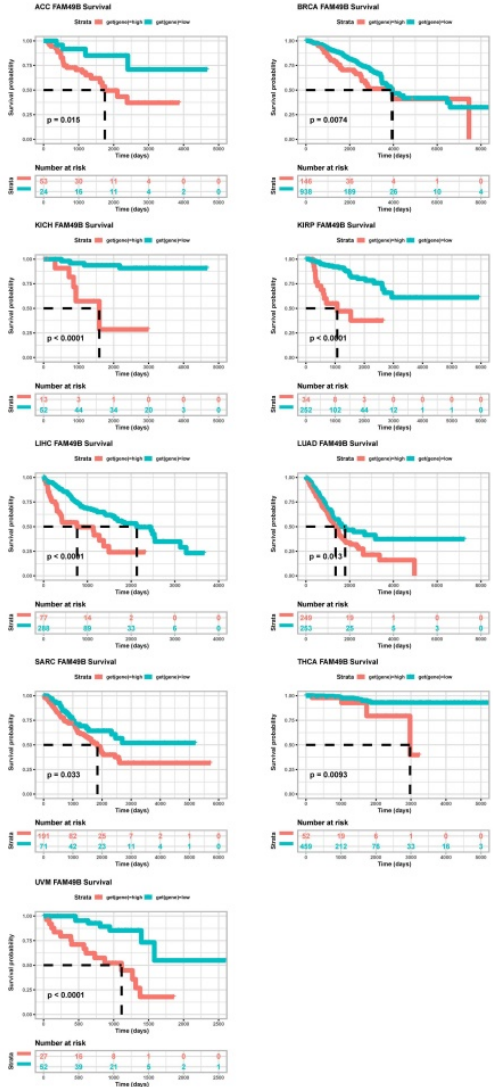
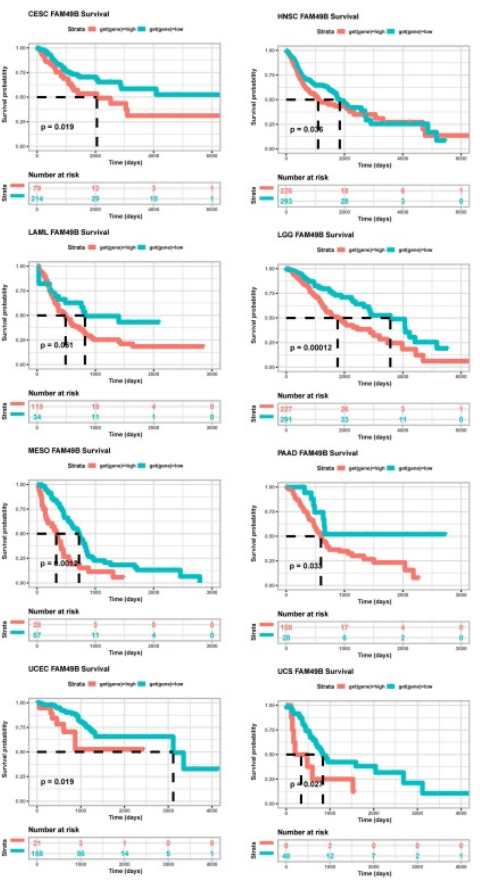

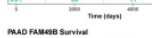
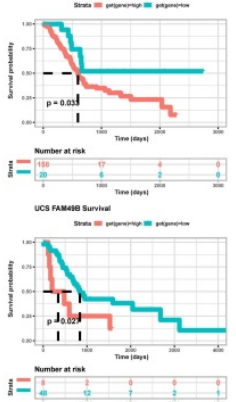

Figure 4. Prognostic significance of FAM49B for OS of patients. (A) The uniCox results of FAM49B in pan-cancer OS of patients. Red colors represent significant results $(p<0.05)$. (B) Kaplan-Meier OS results of FAM49B in pan-cancer. The best cut-off of FAM49B expression was set as cut-off value.
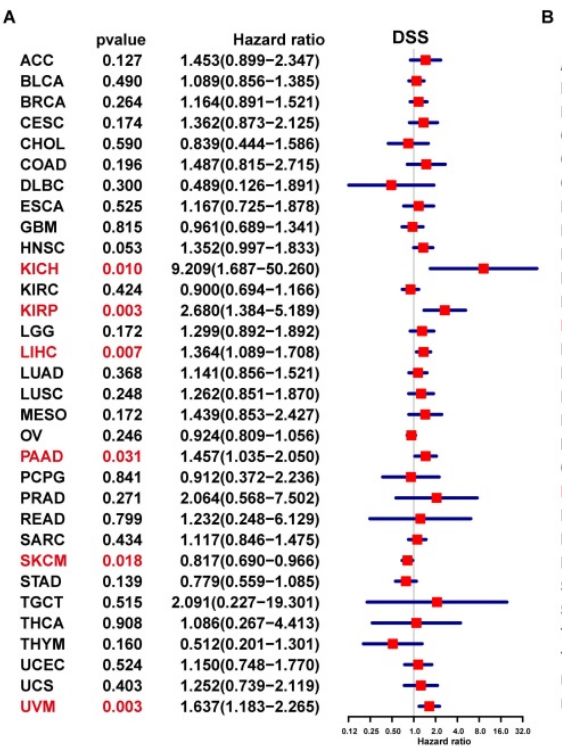

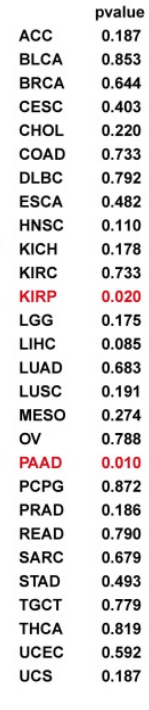

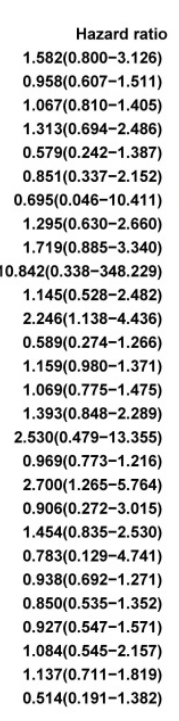

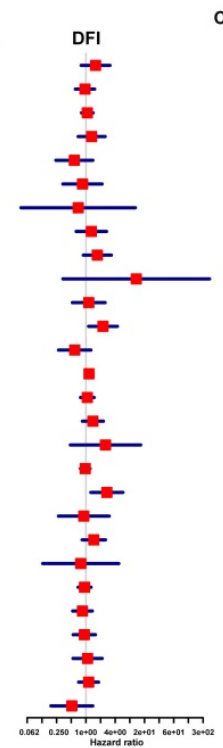

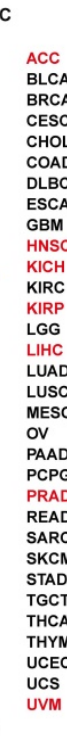
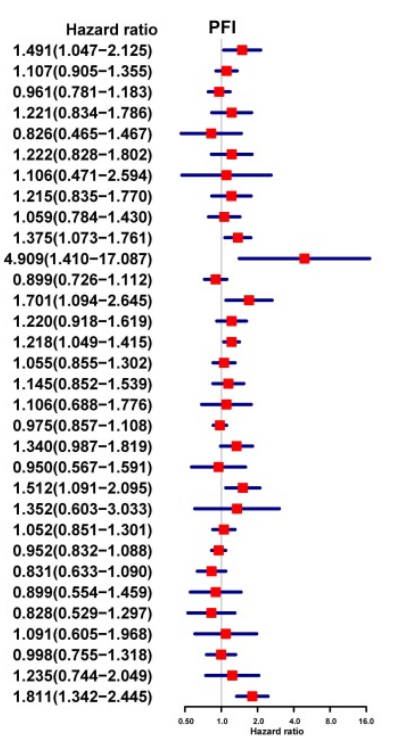

Figure 5. Prognostic significance of FAM49B for DSS, DFI, and PFI of patients. (A-C) The uniCox results of FAM49B in pan-cancer. For DSS (disease-specific survival) (A), DFI (disease-free interval) (B), and PFI (progression-free interval) (C) of patients. Red color represents significant results $(p<0.05)$.

\section{TIME analysis}

To understand the role of FAM49B in TIME of HCC, we performed the correlation analysis between FAM49B expression and stromal and immune scores calculated by $\mathrm{R}$ package "ESTIMATE". Results indicated that FAM49B was positively correlated with stromal score, ESTIMATE score, and immune score and negatively correlated with tumor purity in most tumor types from TCGA (Figure 7A). While for LIHC 
of TCGA, FAM49B was positively associated with immune score and ESTIMATE score and negatively associated with tumor purity score, with no correlation with stromal score (Figure 7B-E).

To prove the immune-regulation function of FAM49B, we downloaded the infiltration level of 24 immune cells from the ImmuCellAI database. Results of the correlation analysis suggested that FAM49B expression was positively correlated with immunosuppressive cells, such as iTreg, Tcm, and nTreg cells, in HCC (Figure 8A).

We also proved that FAM49B expression was positively correlated with immune checkpoints (CTLA4, LAG3, TIGIT, PDCD1, and CD274) in BRCA, HNSC, KICH, KIRP, LIHC, and PAAD (Figure 8B). We further explored the association between FAM49B and immune related genes. As shown in Figure 9, FAM49B was closely associated with MHC genes (Figure 9A), immunosuppressive gene (Figure 9B), immune activated genes (Figure 9C), chemokines (Figure 9D) and chemokine receptors (Figure 9E) in most tumors including HCC. These results suggested that FAM49B play an essential role in tumor immunomodulatory process.

\section{Drug resistance analysis}

Additionally, we analyzed the correlation between FAM49B and IC50 of 192 drugs. Among the 192 anti-cancer drugs, FAM49B expression was positively correlated with IC50 of 8 anti-cancer drugs, such as Ribociclib, Axitinib, GSK269962A, Tozasertib, BMS-754807, and NU7441 (Figure 10A-F). In contrast, FAM49B expression was negatively correlated with IC50 of 132 anti-cancer drugs, such as VE-822, Erlotinib, AZD7762, Ibrutinib, Sapitinib, and Afatinib (Figure 10G-L, Supplementary Table 1).

\section{Methyladenosine (m6A) related genes analysis}

Methyladenosine is the most prevalent reversible methylation in mRNA and has critical roles in the tumorigenesis. The correlation analysis between FAM49B and m6A related genes was performed in pan-cancer. The data showed that FAM49B was positively correlates with m6A related genes in LIHC (Supplementary Figure 3).

\section{Discussion}

Although some progress has been made in cancer-related treatment technology in recent years, the five-year survival rate of HCC is still very low,
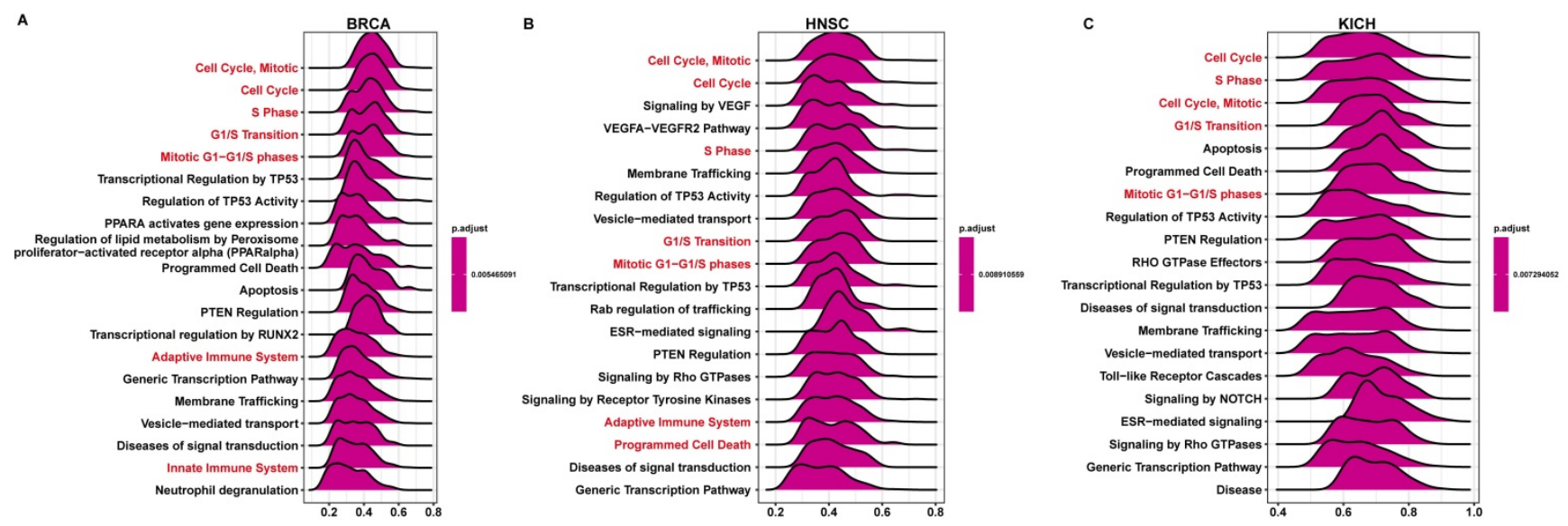

D

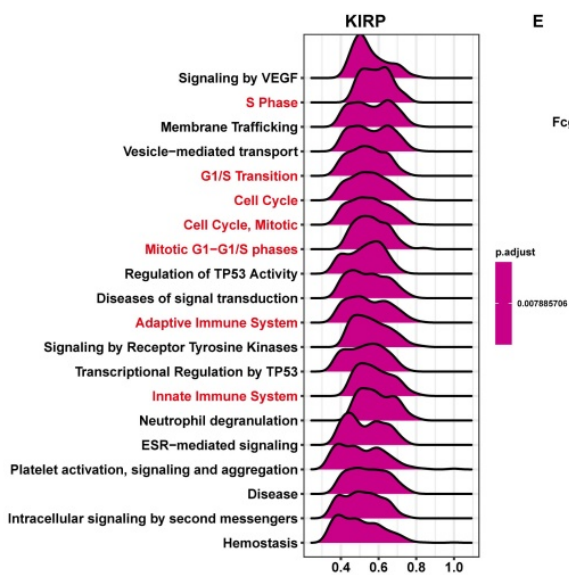

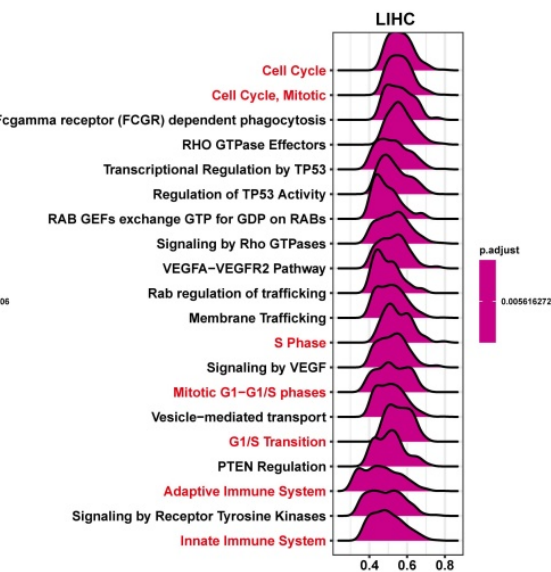

$\mathrm{F}$

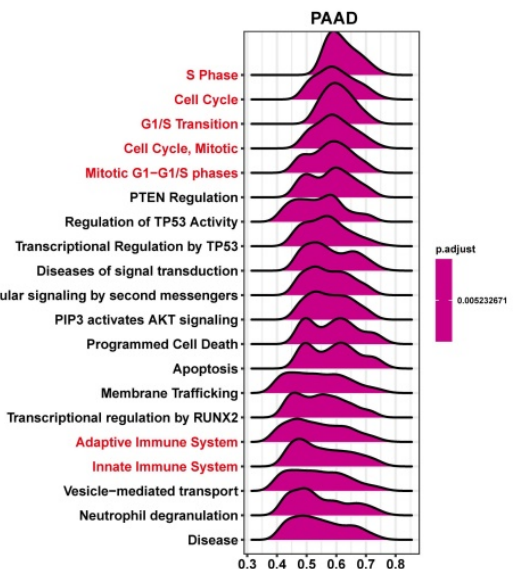

Figure 6. GSEA of FAM49B. (A-F) The top 20 GSEA-Reactome results were showed in indicated tumors. 
mainly because most patients are diagnosed in the final stage [11-13]. Recent studies have shown that the remodeling of TIME by cancer cells plays an important role in the development of HCC, weakening the response of HCC patients to treatment and leading to worse survival status $[14,15]$. Thus, the identification of essential genes that could affect TIME is urgently needed.

FAM49B was previously reported to involve in the progression of several diseases, including tumor [6]. For example, FAM49B was up-regulated and promoted proliferation and metastasis of gallbladder cancer cell [7]. However, its role in HCC remains unclear. In our study, we firstly assessed the expression of FAM49B and found the FAM49B expression was higher in tumor tissues compared with normal tissues in 26 of 31 tumors including HCC. In addition, qRT-PCR assay was used to validate the expression of FAM49B in HCC.

To evaluate the prognostic significance of FAM49B, we performed the uniCox and Kaplan-Meier survival analysis in TCGA cohort. Kaplan-Meier OS analysis proved that an elevated FAM49B expression predicted poorer OS of patients with HCC and other 16 tumors in TCGA cohort. The results of uniCox indicated that FAM49B was a risk factor for the OS, DSS, and PFI of HCC patients.

A

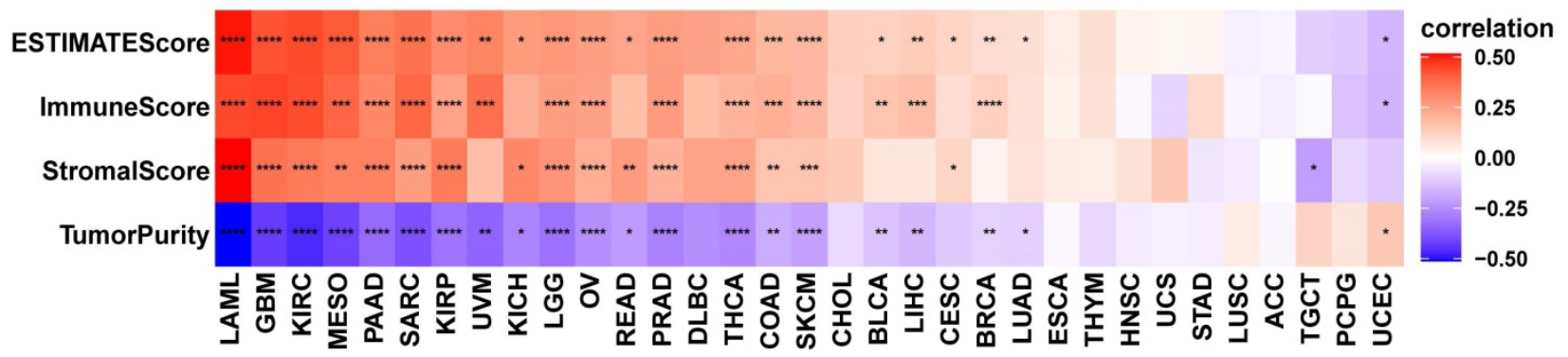

LIHC, $n=372, r=0.17$ (pearson), p.value $=8 e-04$
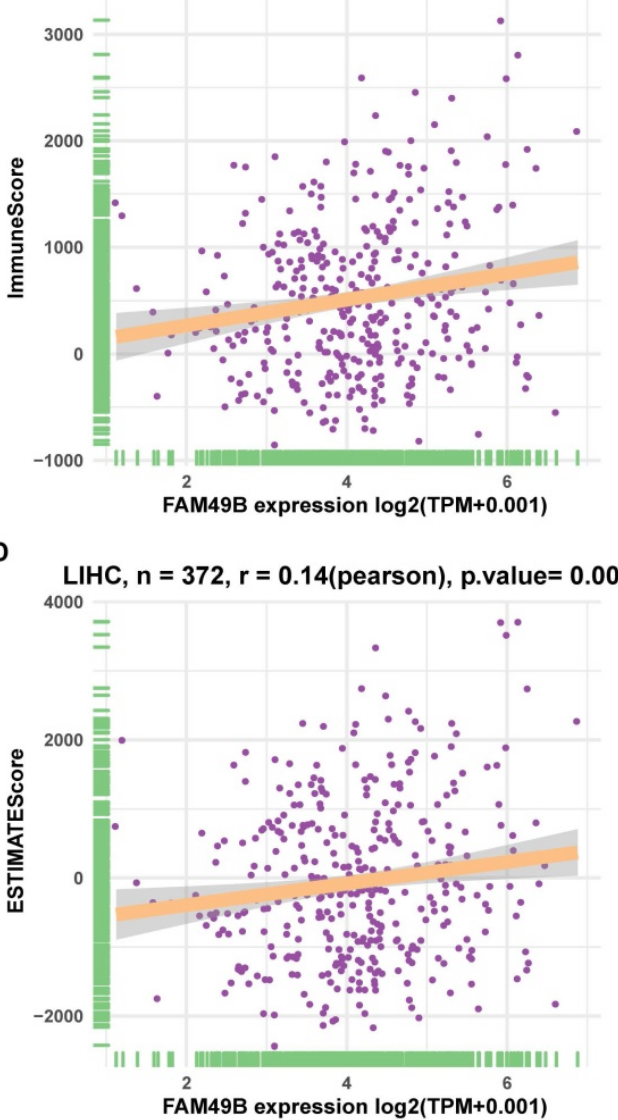

C

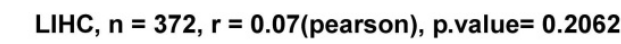

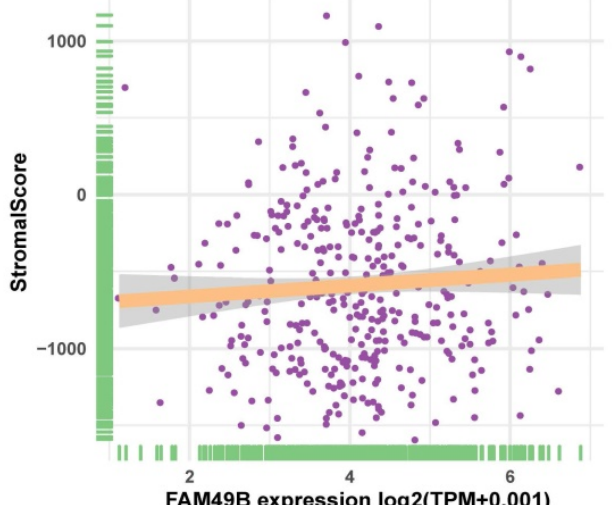

E

LIHC, $n=372, r=-0.16$ (pearson), p.value $=0.0027$

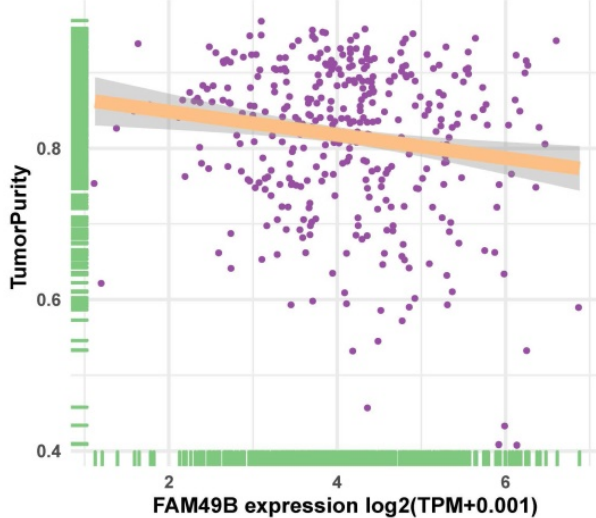

Figure 7. Tumor microenvironment analysis of FAM49B. (A) Heatmap represents the correlation between FAM49B expression and TME scores in pan-cancer. (B-E) The correlation between FAM49B expression and immune score (B), stromal score (C), ESTIMATE score (D), and tumor purity score (E). $*_{p}<0.05$, $* * p<0.01$, $* * * p<0.001$, $* * * * p<0.0001$ 
A

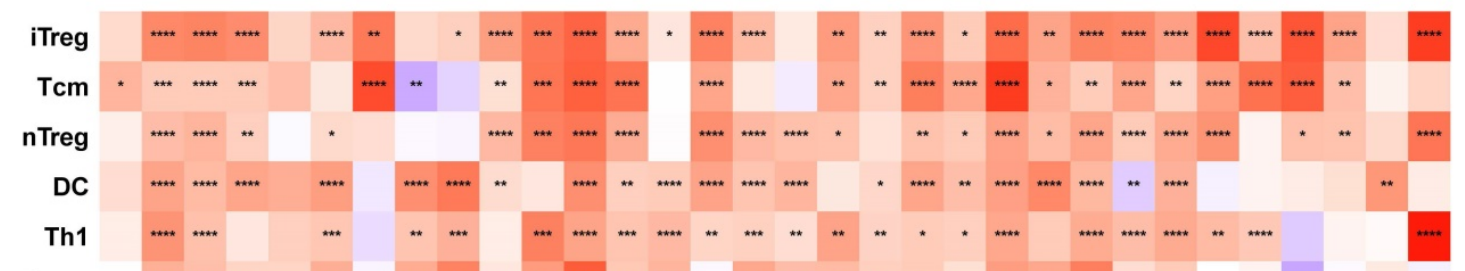

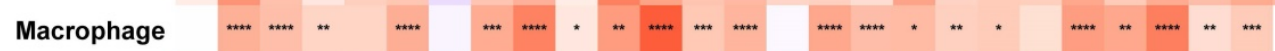

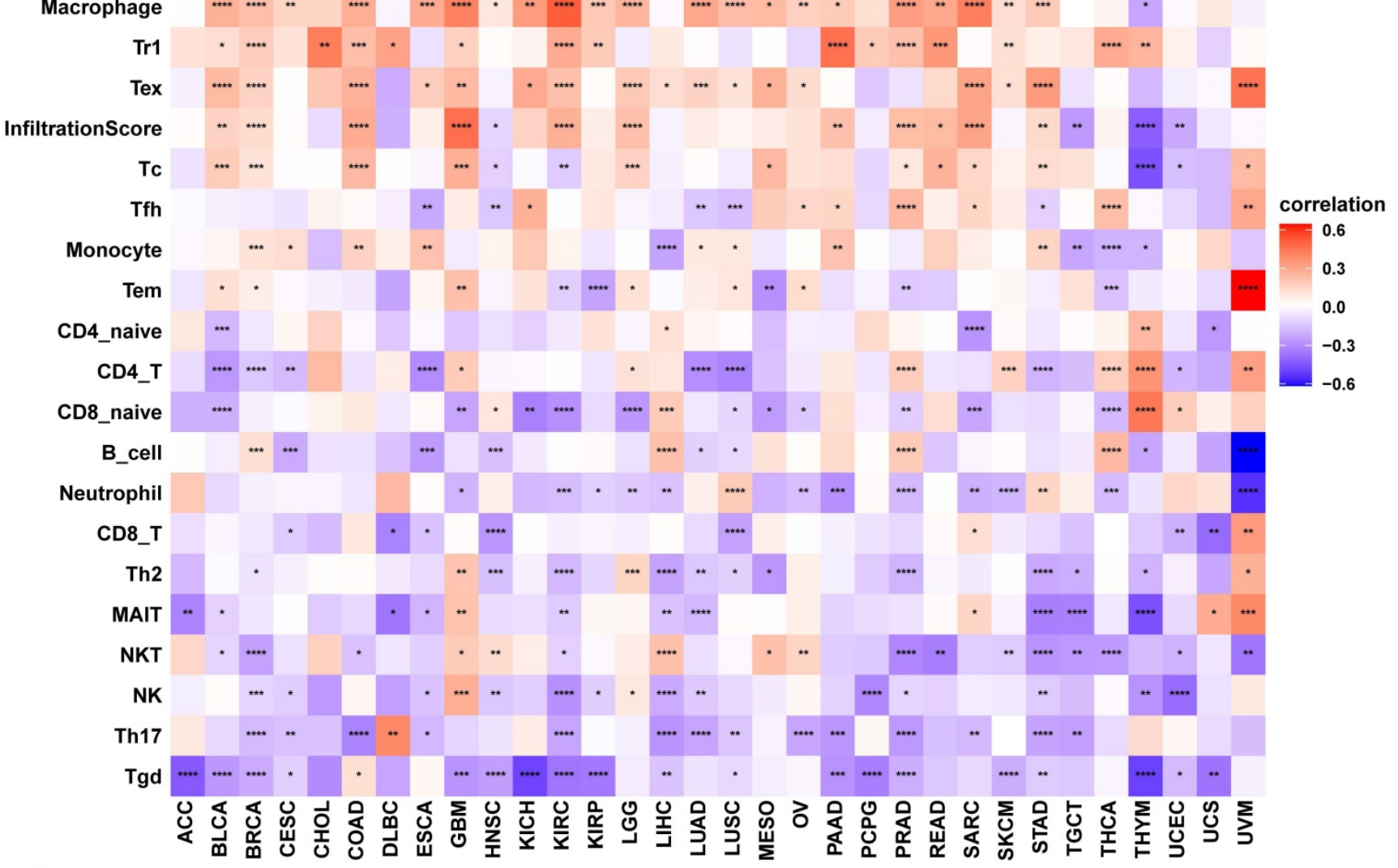

B
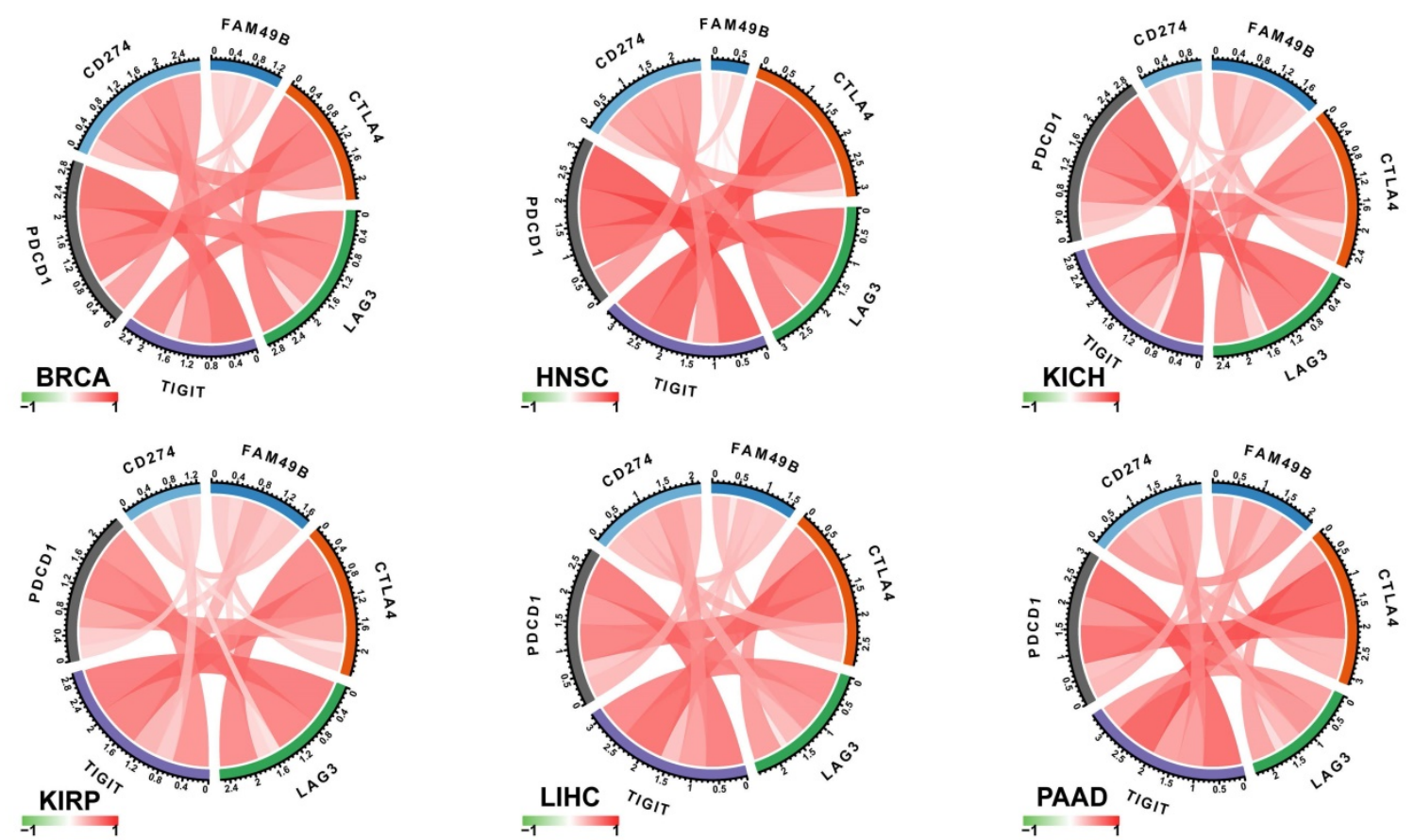

Figure 8. Immune infiltration analysis. (A) The correlation between FAM49B expression and infiltration levels of 24 immune cells. Red represents positive correlation, blue represents negative correlation, and the darker the color, the stronger the correlation. (B) The correlation between FAM49B expression and immune checkpoints in indicated tumors. $* p<0.05, * * p<0.01, * * * p<0.001, * * * * p<0.0001$. 
A

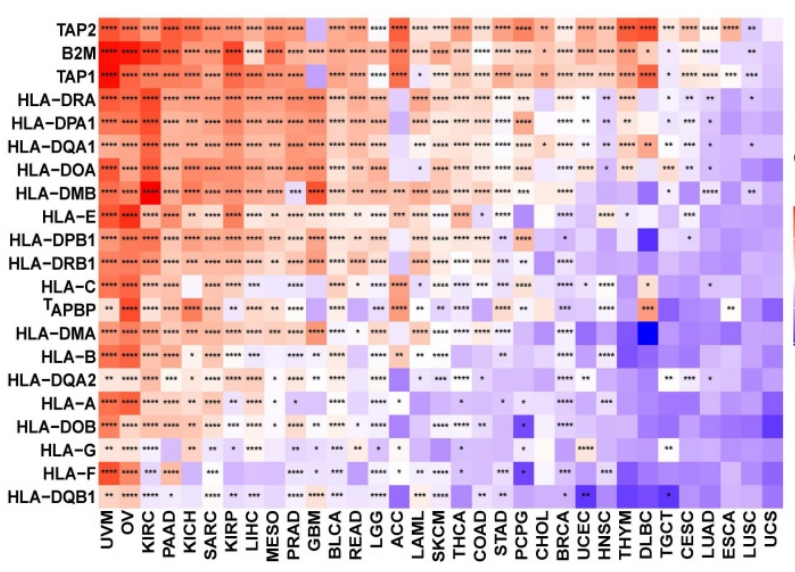

B

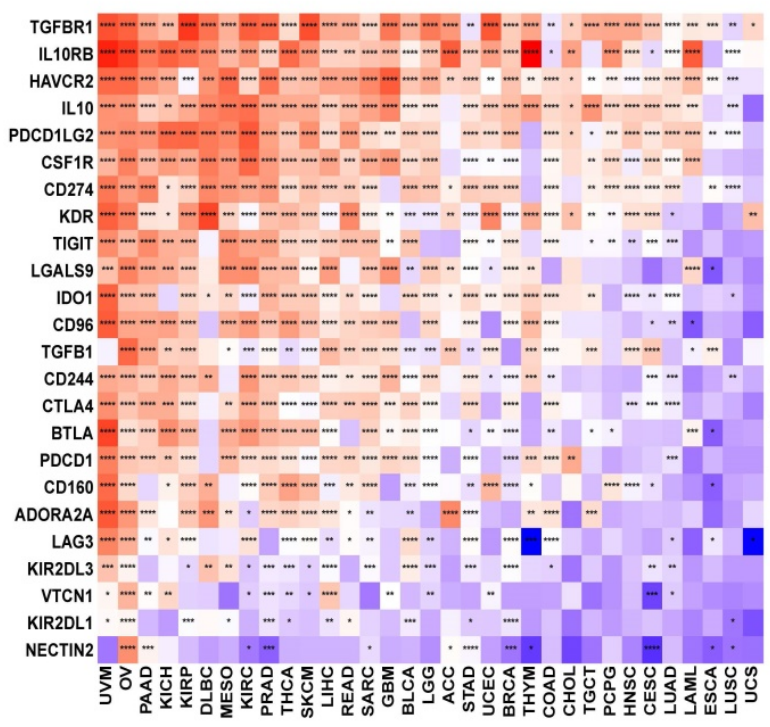

E

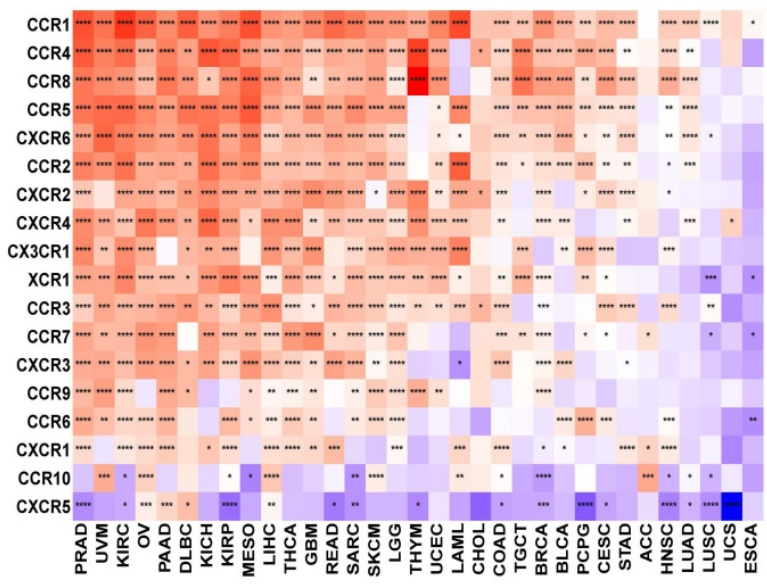

C

${ }_{\text {CD86 }}^{\text {MICB }}$ CDEO

IL2RA

TNFRSF

ENTPD1
CD28
TFS4
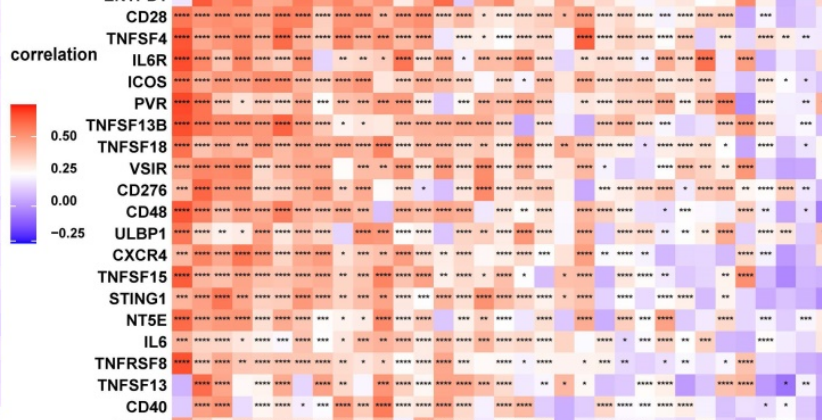

0.50 TNFSF1 138

PVR
ICOS
F13B

0.00

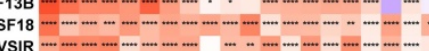

CD276

CD48

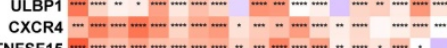

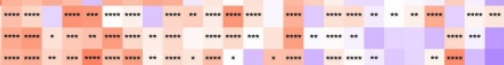

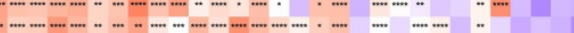

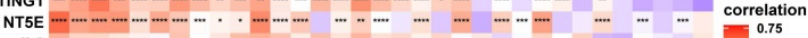

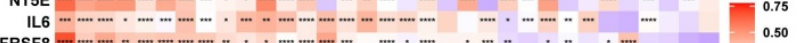

TNFSF13

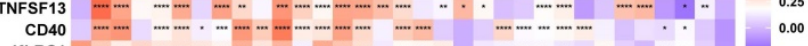

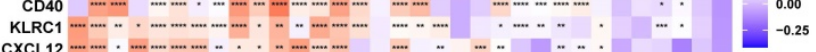

CXCL12 ……....................

CXDLLG

RAET1E

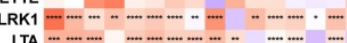

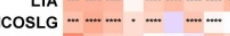

TNFSF9 -

CD27

...........

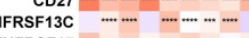

NFRSF

TNFRSF25

HHLA2

TMIGD2
TNFRSF18
TNFRSF4

TNFRSF18
TNFRSF4

TNFRSF18
TNFRSF4
TNFRSF13B
BTNL2
TNFRS14

BTNL2
TNFRSF14

0.25

$0.00 \quad \mathrm{D}$

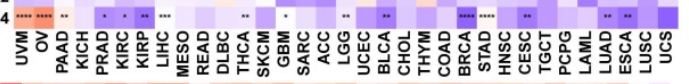

-0.25 CXCL11

CCL11 ………

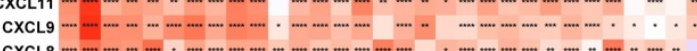

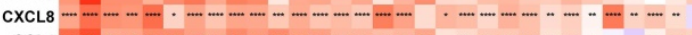

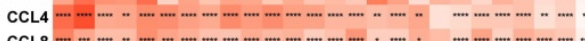

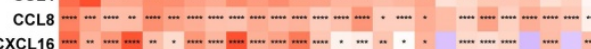

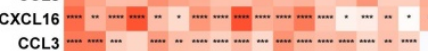

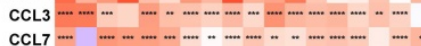

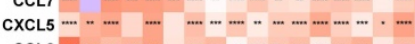

CCL2 ……........................................

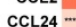

CXCL13

CXCL13
CXCL12

CXCL6 -...

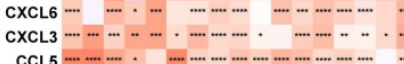

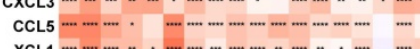

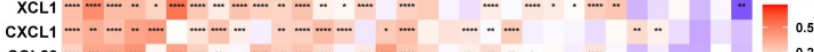

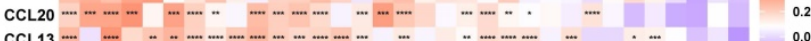

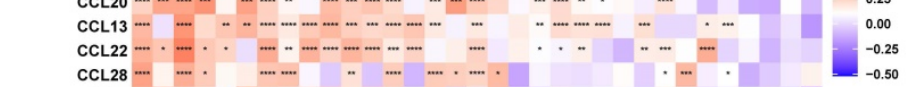

correlation XCL2 -

0.75 CCL23 -...........

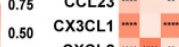

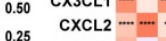

0.25 CCL11 ....

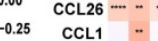

-0.50 CCL19 -..-

CCL21 -

CCL17 -... .

CXCL14 -...

CCL15

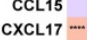

CXCL17
CCL16
CCL27

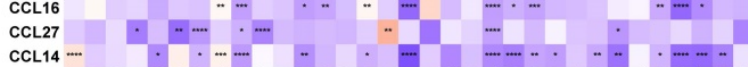

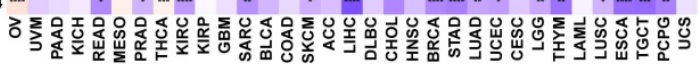

Figure 9. Correlation between immunosuppressive genes. (A) The correlation between FAM49B expression and MHC genes, immunosuppressive genes (B), immune activating genes (C) chemokines (D) and chemokine receptors (E). $*_{p}<0.05, * * p<0.01, * * * p<0.001, * * * * p<0.0001$.

To explore the mechanism of FAM49B affecting prognosis, we performed the GSEA and found that the immune regulation relevant pathways were simultaneously enriched in HCC, indicating an 
essential role of FAM49B TIME. Thus, we further validated the results using immune cell infiltration data from the ImmuCellAI database. Results suggested that FAM49B was positively correlated with immunosuppressive cells, including iTreg, nTreg, and Tcm cells in HCC. In contrast, FAM49B was negatively correlated with immune killer cells, including NK cells in HCC. In addition, we proved that FAM49B expression was positively correlated with immune checkpoints (CTLA4, LAG3, TIGIT, PDCD1, and CD274), MHC genes, immunosuppressive genes, immune activated genes, chemokines, and chemokine receptors in most tumors including HCC. These results suggested that FAM49B play an essential role in tumor immunomodulatory process.
A

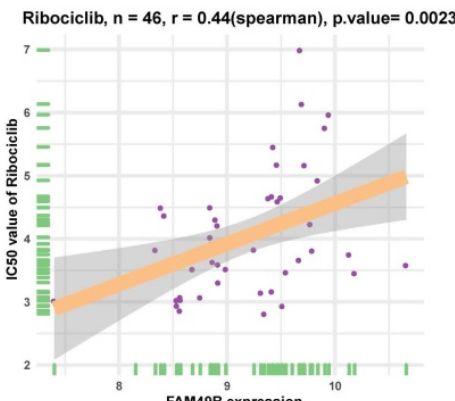

D

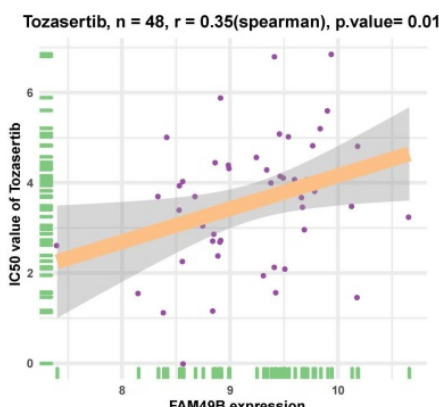

G

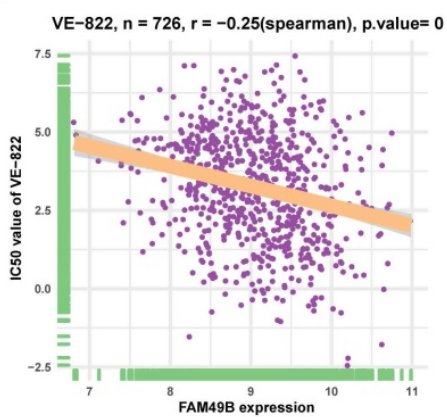

J

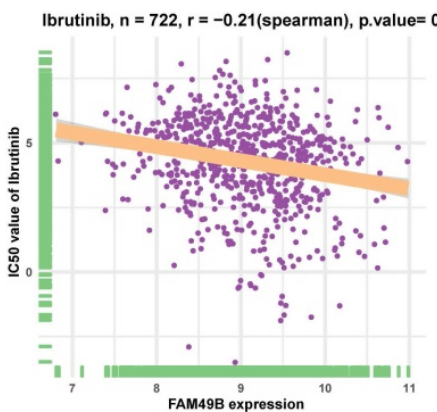

B

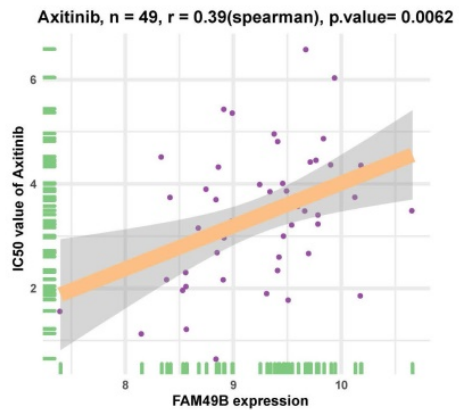

E

BMS-754807, $n=44, r=0.31$ (spearman), p.value $=0.041$

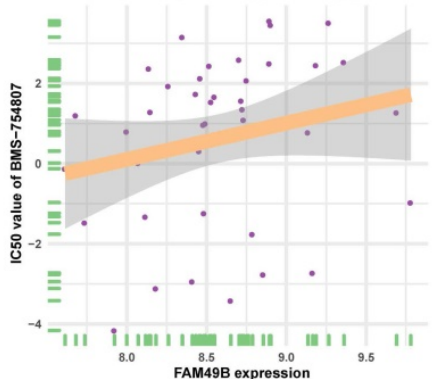

H

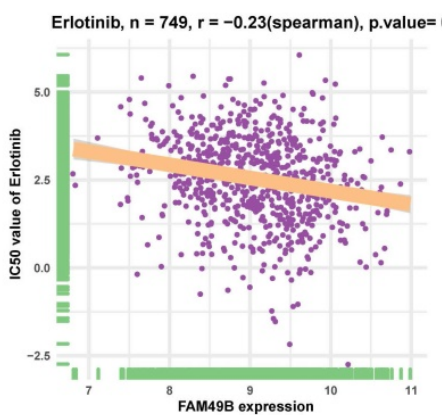

K

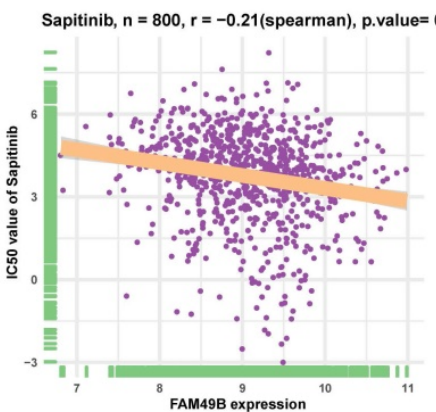

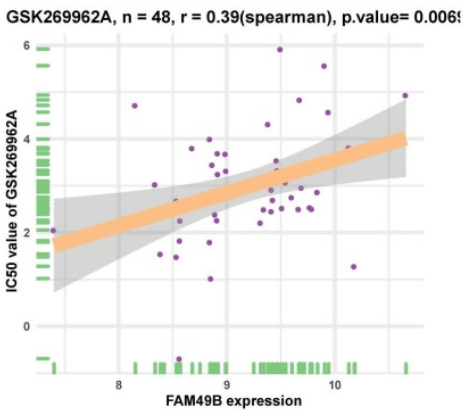

F

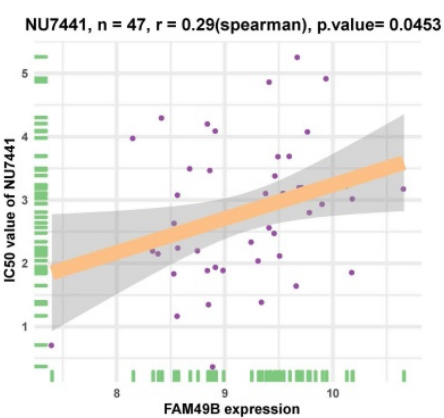

I

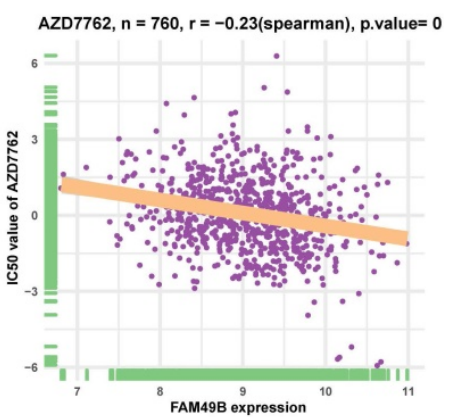

L

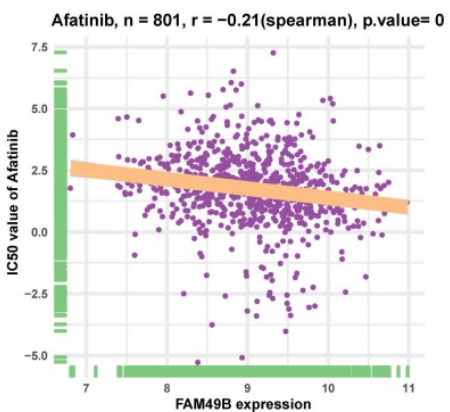

Figure 10. The correlation between FAM49B expression and IC50 values of anti-cancer drugs. (A-L) The correlation between FAM49B expression and IC50 values of indicated anti-cancer drugs. 
Additionally, we also performed the correlation analysis between FAM49B and IC50 of 192 anti-cancer drugs. We found that patients with high FAM49B expression might be resistant to several anti-cancer drugs, such as Ribociclib, Axitinib, GSK269962A, Tozasertib, BMS-754807, and NU7441.

Our findings suggest that FAM49B is a potential prognostic biomarker for HCC. FAM49B play a potential key role in regulating tumor immune microenvironment and anti-tumor drug tolerance.

\section{Abbreviations}

ACC: Adrenocortical carcinoma; BLCA: Bladder Urothelial Carcinoma; BRCA: Breast invasive carcinoma; CESC: Cervical squamous cell carcinoma and endocervical adenocarcinoma; CHOL: Cholangiocarcinoma; COAD: Colon adenocarcinoma; DLBC: Lymphoid Neoplasm Diffuse Large B-cell Lymphoma; ESCA: Esophageal carcinoma; GBM: Glioblastoma multiforme; HNSC: Head and Neck squamous cell carcinoma; $\mathrm{KICH}$ : Kidney Chromophobe; KIRC: Kidney renal clear cell carcinoma; KIRP: Kidney renal papillary cell carcinoma; LAML: Acute Myeloid Leukemia; LGG: Lower Grade Glioma; HCC/LIHC: Liver hepatocellular carcinoma; LUAD: Lung adenocarcinoma; LUSC: Lung squamous cell carcinoma; MESO: Mesothelioma; OV: Ovarian serous cystadenocarcinoma; PAAD: Pancreatic adenocarcinoma; PCPG: Pheochromocytoma and Paraganglioma; PRAD: Prostate adenocarcinoma; READ: Rectum adenocarcinoma; SARC: Sarcoma; SKCM: Skin Cutaneous Melanoma; STAD: Stomach adenocarcinoma; TGCT: Testicular Germ Cell Tumor; THCA: Thyroid carcinoma; THYM: Thymoma; UCEC: Uterine Corpus Endometrial Carcinoma; UCS: Uterine Carcinosarcoma; UVM: Uveal Melanoma; TCGA: The Cancer Genome Atlas; CCLE: Cancer Cell Line Encyclopedia; GTEx: Genotype-Tissue Expression; GDSC: Genomics of Drug Sensitivity in Cancer database; OS: overall survival; DSS: disease-specific survival; DFI: disease-free interval; PFI: progression-free interval.

\section{Supplementary Material}

Supplementary figures.

https://www.jcancer.org/v13p0278s1.pdf

\section{Acknowledgements}

\section{Funding}

This work was supported by the Shaoxing Science and Technology Plan Project (Grant Number 2020A13023, HFW); the Medical Science and Technology Project of Zhejiang Province (Grant
Number 2021RC069).

\section{Competing Interests}

The authors have declared that no competing interest exists.

\section{References}

1. Ioannou GN. HCC surveillance after SVR in patients with F3/F4 fibrosis. J Hepatol. 2021; 74(2): 458-465.

2. Sharma SA, Kowgier M, Hansen BE, Brouwer WP, Maan R, Wong D et al. Toronto HCC risk index: A validated scoring system to predict 10 -year risk of HCC in patients with cirrhosis. J Hepatol. 2017; 24: S0168-8278(17)32248-1.

3. Makarova-Rusher OV, Medina-Echeverz J, Duffy AG, Greten TF. The yin and yang of evasion and immune activation in HCC. J Hepatol. 2015; 62(6):1420-9.

4. Sia D, Jiao Y, Martinez-Quetglas I, Kuchuk O, Villacorta-Martin C, Castro DMM et al. Identification of an Immune-specific Class of Hepatocellular Carcinoma, Based on Molecular Features. Gastroenterology. 2017; 153: 812-826

5. Chen W, Ou M, Tang D, Dai Y, Du W. Identification and Validation of Immune-Related Gene Prognostic Signature for Hepatocellular Carcinoma. J Immunol Res. 2020; 2020: 5494858.

6. Chattaragada MS, Riganti C, Sassoe M, Principe M, Santamorena MM, Roux C et al. FAM49B, a novel regulator of mitochondrial function and integrity that suppresses tumor metastasis. Oncogene. 2018; 37: 697-709.

7. Zhang Y, Du P, Li Y, Zhu Q, Song X, Liu S et al. TASP1 Promotes Gallbladder Cancer Cell Proliferation and Metastasis by Up-regulating FAM49B via PI3K/AKT Pathway. Int J Biol Sci. 2020; 16: 739-751.

8. Geng $\mathrm{Q}$, Shen Z, Li L, Zhao J. COL1A1 is a prognostic biomarker and correlated with immune infiltrates in lung cancer. Peer J. 2021; 9: e11145.

9. Shang W, Jiang Y, Boettcher M, Ding K, Mollenauer M, Liu Z et al. Genomewide CRISPR screen identifies FAM49B as a key regulator of actin dynamics and T cell activation. Proc Natl Acad Sci USA. 2018; 115: E4051-E4060.

10. The Cancer Genome Atlas Research Network, David A. Wheeler, and Lewis R. Roberts. Comprehensive and Integrative Genomic Characterization of Hepatocellular Carcinoma. Cell. 2017; 15; 169(7): 1327-1341.e23.

11. Wong RJ, Ahmed A. Understanding Gaps in the Hepatocellular Carcinoma Cascade of Care: Opportunities to Improve Hepatocellular Carcinoma Outcomes. J Clin Gastroenterol. 2020; 54: 850-856.

12. Jiang YF, Yang ZH, Hu JQ. Recurrence or metastasis of HCC: predictors, early detection and experimental antiangiogenic therapy. World J Gastroenterol. 2000; 6: 61-65.

13. Liu C, Zhou X, Long Q, Zeng H, Sun Q, Chen $Y$ et al. Small extracellular vesicles containing miR-30a-3p attenuate the migration and invasion of hepatocellular carcinoma by targeting SNAP23 gene. Oncogene. 2021; 40: 233-245.

14. Hong W, Liang L, Gu Y, Qi Z, Qiu H, Yang X et al. Immune-Related IncRNA to Construct Novel Signature and Predict the Immune Landscape of Human Hepatocellular Carcinoma. Mol Ther Nucleic Acids. 2020; 22: 937-947.

15. Kurebayashi Y, Ojima H, Tsujikawa H, Kubota N, Maehara J, Abe Y et al. Landscape of immune microenvironment in hepatocellular carcinoma and its additional impact on histological and molecular classification. Hepatology. 2018; 68: 1025-1041. 